\title{
AN INVARIANT APPROACH TO THE THEORY OF LOGARITHMIC KODAIRA DIMENSION OF ALGEBRAIC VARIETIES
}

\author{
ZHAOHUA LUO
}

Let $V$ be an algebraic variety defined over a field $k$. If $K$ is the rational function field of $V$, then $V$ is called a model of $K / k$, and the local ring of a point of $V$ is a locality of $V$. Let $L(K / k)$ be the set of discrete valuation rings of $K / k$. Define

$$
\begin{aligned}
& \tilde{L}(V)=\{R \in L(K / k) \mid R \text { dominates a locality of } V\}, \\
& L(V)=\{R \in L(K / k) \mid R \text { is a locality of the normalization } \bar{V} \text { of } V\} .
\end{aligned}
$$

If $V^{\prime}$ is another model of $K / k$ and $\tilde{L}(V)=\tilde{L}\left(V^{\prime}\right)$, then we say that $V$ and $V^{\prime}$ are proper birationally equivalent. The logarithmic Kodaira dimension $\kappa(V)$ of $V$ introduced by litaka (see [1]) is one of the most important proper birational invariants of $V$. Iitaka's treatment requires Hironaka's theory of resolution of singularities, and therefore at present does not apply to the cases of positive characteristics. In this note we shall describe a simple invariant approach to the theory of logarithmic Kodaira dimension of algebraic varieties defined over an arbitrary base field.

I would like to thank Professor T. Matsusaka for his encouragement throughout the stages of development of these results.

1. A divisor of $K / k$ is by definition a map $w: L(K / k) \rightarrow \mathbf{Z} \cup\{+\infty\}$ such that $w^{-1}(\mathbf{Z}-\{0\}) \cap L(V)$ is a finite set for one (therefore for any) model $V$ of $K / k ; w$ is called absolute if $w(L(K / k)) \subseteq \mathbf{Z}$; it is called effective (denoted by $w \geq 0)$ if $w(R) \geq 0$ for all $R \in L(K / k)$. For any $u \in K$ we define the principal divisor $(u)_{K / k}$ of $K / k$ by $(u)_{K / k}(R)=v_{R}(u)$ for all $R \in L(K / k)$, where $v_{R}$ is the normalized discrete valuation of $K / k$ determined by $R \in L(K / k)$. The divisors of $K / k$ form an abelian semigroup under pointwise addition. Two divisors $w$ and $w^{\prime}$ of $K / k$ are linearly equivalent (notation: $w \sim w^{\prime}$ ) if $w=w^{\prime}+(u)_{K / k}$ for some $u \in K$.

Let $V$ be a model of $K / k$. We define two divisors $S_{V}$ and $T_{V}$ of $L(K / k)$ by the following rules:

$$
\left\{\begin{array} { l l } 
{ S _ { V } ( R ) = 0 } & { \text { for } R \in \tilde { L } ( V ) , } \\
{ S _ { V } ( R ) = + 1 } & { \text { for } R \notin \tilde { L } ( V ) }
\end{array} \quad \left\{\begin{array}{ll}
T_{V}(R)=0 & \text { for } R \in L(V), \\
T_{V}(R)=+\infty & \text { for } R \notin L(V) .
\end{array}\right.\right.
$$

If $w$ is a divisor of $K / k$ we define

$$
\tilde{w}_{V}=w+S_{V}, \quad w_{V}=w+T_{V} .
$$

Received by the editors December 22, 1986.

1980 Mathematics Subject Classification (1985 Revision). Primary 14J99; Secondary $14 \mathrm{C} 20$. 
Let $\Gamma(K / k)$ be the set of pairs $\left(R, R^{\prime}\right)$ of regular localities of $K / k$ such that $R$ dominates $R^{\prime} \subset R$ and krull $\operatorname{dim} R=1$. Any absolute divisor $w$ of $K / k$ determines a map $r_{w}: \Gamma(K / k) \rightarrow \mathbf{Z}$ by

$$
r_{w}\left(R, R^{\prime}\right)=w(R)-v_{R}(u)
$$

where $u$ is a local function of $w$ at $R^{\prime}$ (i.e. $v_{R^{\prime \prime}}(u)=w\left(R^{\prime \prime}\right)$ for any $R^{\prime \prime} \in$ $\left.L\left(\operatorname{spec}\left(R^{\prime}\right)\right)\right)$. We call $r_{w}$ the ramification index of $K / k$ determined by $w$.

An absolute divisor $w$ of $K / k$ is called proper birationally invariant if for any $\left(R, R^{\prime}\right) \in \Gamma(K / k)$ we have $r_{w}\left(R, R^{\prime}\right) \geq \operatorname{krull} \operatorname{dim} R^{\prime}-1$.

Given any dominating pair $\left(R, R^{\prime}\right)$ of regular local rings such that

\section{krull $\operatorname{dim} R=1$}

and the quotient field of $R$ is a finite separable extension of the quotient field of $R^{\prime}$, we introduce two invariants of $\left(R, R^{\prime}\right)$ :

$r\left(R, R^{\prime}\right)=v_{R}\left(d\left(R / R^{\prime}\right)\right)$, where $d\left(R / R^{\prime}\right)$ is the Kähler different of $R$ over $R^{\prime}$

$e\left(R, R^{\prime}\right)=\max \left\{v_{R}\left(u_{1}, \ldots, u_{r}\right) \mid\left(u_{1}, \ldots, u_{r}\right)\right.$ is a minimal basis of the maximal ideal of $\left.R^{\prime}\right\}$.

The integers $r\left(R, R^{\prime}\right)$ and $e\left(R, R^{\prime}\right)$ are called the ramification index and the reduced ramification index of $\left(R, R^{\prime}\right)$ respectively.

In case that krull $\operatorname{dim} R^{\prime}=1$ we have $r\left(R, R^{\prime}\right) \geq e\left(R, R^{\prime}\right)-1$ by the main theorem of ramification theory of algebraic number theory due to Dedekind. In [3] we proved that this is true in general, i.e.,

$$
r\left(R, R^{\prime}\right) \geq e\left(R, R^{\prime}\right)-1 \geq \text { krull } \operatorname{dim} R^{\prime}-1 .
$$

(see [4] for an application of this formula).

Now back to our birational situation. We have the following theorem.

THEOREM 1.1. If $w$ is a proper birationally invariant divisor of $K / k$, then $r_{w}\left(R, R^{\prime}\right) \geq r\left(R, R^{\prime}\right) \geq e\left(R, R^{\prime}\right)-1$ for any $\left(R, R^{\prime}\right) \in \Gamma(K / k)$.

2. We shall fix a polynomial ring $A=\bigoplus_{i=0}^{\infty} A_{i}=K[X]$ in one variable $X$ over $K$. For any divisor $w$ of $K / k$ and $m=u X^{i} \in A_{i}$ we let $w(m)=$ $(u)_{K / k}+i w$ (we assume $\left.0 \cdot(+\infty)=+\infty\right)$; put $C_{i}(w)=\left\{m \in A_{i} \mid w(m) \geq 0\right\}$, $C(w)=\bigoplus_{i=0}^{\infty} C_{i}(w), Z(w)=Q C(w) \cap K$ where $Q C(w)$ is the quotient field of $C(w)$, and $\kappa(w)=$ trans. $\operatorname{deg} C(w) / k-1$. Define $\bar{Z}(w)=\bigcap Z\left(w_{V}\right)$ and $\bar{\kappa}=\min \kappa\left(w_{V}\right)$, where $V$ runs through the set of models of $K / k$. One can prove the following theorem easily (cf. [2]).

THEOREM 2.1. If $w$ and $w^{\prime}$ are linearly equivalent divisors of $K / k$ then $C(w) \cong C\left(w^{\prime}\right) ; C(w)$ is an integrally closed $k$-graded algebra; $Z(w)$ and $\bar{Z}(w)$ are algebraically closed in $K$; if $w$ is absolute, then $\operatorname{dim}_{k} C_{i}<+\infty$.

Suppose $X$ is a model of $K / k$ and $D$ a reduced divisor of the normalization $\bar{X}$ of $X$. If $\tilde{L}(V)=\tilde{L}(\bar{X}-\sup D)$, then the pair $(X, D)$ is called a model of $V$; if $D=0$ we also say that $X$ is a model of $V$. A model $(X, D)$ of $V$ is regular if $X$ is nonsingular, $D$ is a sum of nonsingular subvarieties and $\sup D$ has only normal crossings. 
With the help of Theorem 1.1 we can prove the following

THEOREM 2.2. If $w$ is a proper birationally invariant absolute divisor of $K / k$ and $(X, D)$ a regular complete model of a model $V$ of $K / k$, then

$$
C\left(\widetilde{w_{V}}\right)=C\left(\left(\widetilde{w_{V}}\right)_{X}\right)=\bigoplus_{i=0}^{\infty} H^{0}\left(X, O_{X}(i(w(X)+D)),\right.
$$

where $w(X)=\left.w\right|_{L(X)}$ is the Weil divisor of $X$ induced by $w, Z\left(\widetilde{w_{V}}\right)=$ $\bar{Z}\left(\widetilde{w_{V}}\right)=Z\left(\left(\widetilde{w_{V}}\right)_{X}\right)$ and $\kappa\left(\widetilde{w_{V}}\right)=\bar{\kappa}\left(\widetilde{w_{V}}\right)=\kappa\left(\left(\widetilde{w_{V}}\right)_{X}\right)$.

ProOF. It suffices to prove that $C\left(\tilde{w}_{V}\right)=C\left(\left(\tilde{w}_{V}\right)_{X}\right)$ because then all the other assertions follow by definitions. Since $\tilde{w}_{V} \leq\left(\tilde{w}_{V}\right)_{X}, C\left(\tilde{w}_{V}\right) \subseteq$ $C\left(\left(\tilde{w}_{V}\right)_{X}\right)$, so we only need to prove $C\left(\left(\tilde{w}_{V}\right)_{X}\right) \subseteq C\left(\tilde{w}_{V}\right)$.

To simplify notations we write $w^{\prime}$ for $\tilde{w}_{V}$ and $w^{\prime \prime}$ for $\left(\tilde{w}_{V}\right)_{X}$.

For any $R \in L(K / k)$ let $R^{\prime}$ be the local ring of the center $P$ of $v_{R}$ on $X$. Then $P \in \sup D$ if and only if $R \notin \tilde{L}(V)$. Let $\left(u_{1}, \ldots, u_{r}\right)$ be a minimal basis of the maximal ideal of $R^{\prime}$ such that, if $P \in \sup D,\left(u_{1}, \ldots, u_{t}\right)$ is a set of local equations of the divisor $D$ at $P$ for some $1 \leq t \leq r$. Write $a=u_{1} \cdots u_{r}$ and $b=u_{1} \cdots u_{t}$ (if $R \in \tilde{L}(V)$ we let $b=1$ ). Let $u^{\prime}$ be a local function of $w$ at $R^{\prime}$.

Now suppose $m=u X^{i} \in C_{i}\left(w^{\prime \prime}\right)$. We have to prove that $m \in C_{i}\left(w^{\prime}\right)$, i.e., $w^{\prime}(m)(R) \geq 0$ for any $R \in L(K / k)$. For any $R^{\prime \prime} \in L\left(\operatorname{spec}\left(R^{\prime}\right)\right)$ we have $w^{\prime \prime}(m)\left(R^{\prime \prime}\right)=v_{R^{\prime \prime}}\left(u\left(u^{\prime} b\right)^{i}\right) \geq 0$, which implies that $u\left(u^{\prime} b\right)^{i} \in \bigcap R^{\prime \prime}=R^{\prime} \subseteq R$. It follows that $v_{R}(u)+i\left(v_{R}\left(u^{\prime}\right)+v_{R}(b)\right) \geq 0$. But $w(R)-v_{R}\left(u^{\prime}\right) \geq v_{R}(a)-1$ by Theorem 1.1. Hence

$$
v_{R}(u)+i\left(w(R)+1-v_{R}(a)+v_{R}(b)\right) \geq 0 .
$$

Now recall the definition of $w^{\prime}(m)(R)$ :

$$
\begin{cases}w^{\prime}(m)(R)=v_{R}(u)+i(w(R)+1) & \text { for } R \notin \tilde{L}(V), \\ w^{\prime}(m)(R)=v_{R}(u)+i(w(R)) & \text { for } R \in \tilde{L}(V) .\end{cases}
$$

If $R \notin \tilde{L}(V)$ then $w^{\prime}(m)(R) \geq 0$ because in $(*)-v_{R}(a)+v_{R}(b) \leq 0$. If $R \in$ $\tilde{L}(V)$ then $w^{\prime}(m)(R) \geq 0$ because in $(*) v_{R}(b)=v_{R}(1)=0$ and $1-v_{R}(a) \leq 0$. Thus we have proved that $w^{\prime}(m)(R) \geq 0$ for any $R \in L(K / k)$. This finishes the proof.

3. Let $k^{\prime}$ be a perfect subfield of $k$ (e.g., the prime field of $k$ ) and $D\left(K / k^{\prime}\right)$ the differential module of $K$ over $k^{\prime}$. A subset $B$ of $K$ is called a $k^{\prime}$-differential basis of $K / k$ if $d B$ is a $K$-linear basis for $D\left(K / k^{\prime}\right)$ and $B-B \cap k$ is a finite set. If $R \in L(K / k)$ we proved in [2] that $R d R$ is an $R$-free module and there exists a $k^{\prime}$-differential basis $B_{R}$ of $K / k$ such that $d B_{R}$ is an $R$-free basis for $R d R ; B_{R}$ is called a set of $k^{\prime}$-uniformizing coordinates of $R$.

For any two $k^{\prime}$-differential bases $B, B^{\prime}$ of $K / k$ one can define an element $J\left(B, B^{\prime}\right) \in K$, uniquely determined by $\left(B, B^{\prime}\right)$ up to a factor in the algebraic closure $\bar{k}$ of $k$ in $K$, such that, if $B, B^{\prime}$ are two sets of $k^{\prime}$-uniformizing coordinates for some $R \in L(K / k)$, then $J\left(B, B^{\prime}\right)$ is an invertible element of $R$.

For any $k^{\prime}$-differential basis $B$ of $K / k$ we define the divisor $(B)$ of $K / k$ by $(B)(R)=v_{R}\left(J\left(B, B_{R}\right)\right)$, where $B_{R}$ is a set of $k^{\prime}$-uniformizing coordinates of $R$. 
If $k^{\prime \prime}$ is another perfect subfield of $k$ and $B^{\prime \prime}$ a $k^{\prime \prime}$-differential basis of $K / k$, then one can show that $(B)$ and $\left(B^{\prime \prime}\right)$ are linearly equivalent. Any divisor of $K / k$ which is linearly equivalent to $(B)$ is called a canonical divisor of $K / k$. We summarize the main properties of the canonical divisors of $K / k$ in the following theorem.

THEOREM 3.1. (1) If $F$ is a subfield of $K$ containing $k$, then $\left.(B)\right|_{L(K / F)}$ is a canonical divisor of $K / F ;(2)(B)$ is proper birationally invariant.

4. Let $V$ be a model of $K / k$ and $w$ a canonical divisor of $K / k$. It is easy to see that $C\left(\tilde{w}_{V}\right), Z\left(\tilde{w}_{V}\right), \bar{Z}\left(\tilde{w}_{V}\right), \kappa\left(\tilde{w}_{V}\right), \bar{\kappa}\left(\tilde{w}_{V}\right)$ are proper birational invariants of $V$, denoted by $C(V), Z(V), \bar{Z}(V), \kappa(V), \bar{\kappa}(V)$ respectively. If $V$ is complete, then $\tilde{w}_{V}=w$, therefore $C(V), Z(V), \ldots$ are all birational invariants of $V$, denoted by $C(K / k), Z(K / k), \ldots$ respectively.

DEFINITION 4.1. $\kappa(V)$ and $\kappa(K / k)$ are called the (logarithmic) Kodaira dimension of $V$ and $K / k$ respectively; $\bar{\kappa}(V)$ and $\bar{\kappa}(K / k)$ are the virtual (logarithmic) Kodaira dimension of $V$ and $K$ respectively.

Since canonical divisors of $K / k$ are proper birationally invariant, we see from Theorem 2.2 that our definition of $\kappa(V)$ is equivalent to that of Iitaka's whenever the latter is applicable (notice that $\kappa(V)$ is usually denoted by $\tilde{\kappa}(V))$.

Let $F$ be a subfield of $K$ containing $k$. If $U=\operatorname{spec} B$ is an affine open subset of $V$, then $U^{\prime}=\operatorname{spec} F(B)$ is an affine model of $K / F$, here $F(B)$ is the affine ring of $K / F$ generated by the affine $\operatorname{ring} B$ over $F$. The collection of all such $U^{\prime}$ defines a model $V_{K / F}$ of $K / F$. Applying Theorem 3.1(1) we can prove the following

THEOREM 4.2. (1) If $\bar{\kappa}(V) \geq 0$ then $\bar{\kappa}\left(V_{K / \bar{Z}(V)}\right)=0$; (2) If $\bar{\kappa}\left(V_{K / F}\right)=0$ then $F \supseteq \bar{Z}(V) ;(3) \kappa(V) \leq \kappa\left(V_{K / F}\right)+\operatorname{dim} F / k$ and $\bar{\kappa}(V) \leq \bar{\kappa}\left(V_{K / F}\right)+$ $\operatorname{dim} F / k$.

THEOREM 4.3. Any $K / k$ can be uniquely factored into a series of extensions: $k \subseteq \bar{k}=F_{0} \varsubsetneqq F_{1} \varsubsetneqq F_{2} \cdots \varsubsetneqq F_{r-1} \varsubsetneqq F_{r}=K, 0 \leq r \leq \operatorname{dim} K / k$ such that (1) every $F_{i}$ is algebraically closed in $K ;(2) \bar{\kappa}\left(F_{1} / F_{0}\right) \leq 0$ or $\bar{\kappa}\left(F_{1} / F_{0}\right)=$ $\operatorname{dim} F_{1} / F_{0} ;(3) \bar{\kappa}\left(F_{i} / F_{i-1}\right)=0$ for $1<i \leq r ;(4) \bar{\kappa}\left(F_{i} / F_{0}\right)=\operatorname{dim} F_{i-1} / F_{0}$ for $1<i \leq r$.

When $\operatorname{ch} k=p>0$ for geometric reasons it is important to know whether $K / Z(K / k)$ is a regular extension in the case that $0<\kappa(K / k)<\operatorname{dim} K / k$. In this respect we have the following.

THEOREM 4.4. Suppose $p \neq 2,3$, and $\bar{\kappa}(K / k)=\operatorname{dim} K / k-1$. Then $K / \bar{Z}(K / k)$ is a regular extension.

PrOOF. We have $\operatorname{dim} K / \bar{Z}(K / k)=1$ and $\kappa(K / \bar{Z}(K / k))=\bar{\kappa}(K / \bar{Z}(K / k))$ $=0$ by Theorem 4.3. Thus the genus of $K / \bar{Z}(K / k)$ is 1 . According to [5], the genus $g$ of an inseparable algebraic function field of one variable of characteristic $p>0$ satisfies the relation $2 g \geq p(p-3)+2$. In our case $g=1$ and $p \neq 2,3$. It is immediate that $K / \bar{Z}(K / k)$ must be a separably generated extension, hence a regular extension. 
COROLlaRY 4.5. Suppose $k$ is perfect and $p \neq 2,3$, and $\operatorname{dim} K / k \leq 3$. Then $K / \bar{Z}(K / k)$ is separably generated.

5. Let $V$ be a model of $K / k$ and $P(V)=\left\{f \in\right.$ Aut $_{k} K \mid$ the map $f^{\prime}: L(K / k) \rightarrow L(K / k)$ induced by $f$ maps $L(V)$ onto $\left.L(V)\right\}$. If $V=\operatorname{spec} B$ is a normal affine model of $K / k$ then $B=\bigcap_{R \in \tilde{L}(V)} R$, hence $f \in P(V)$ if and only if $f(B)=B$; therefore $P(V)=\operatorname{Aut}_{k}(B)$.

THEOREM 5.1. Assume $k$ is algebraically closed. (1) If $\kappa(K / k)=$ $\operatorname{dim} K / k$, then Aut $_{k} K$ is a finite group. (2) If $\kappa(V)=\operatorname{dim} V$, then $P(V)$ is a finite group. (3) Suppose $V=\operatorname{spec} B$ and $V$ has a regular complete model. If $\kappa(K / k) \geq 0$, then $P(V)$ is a finite group.

The assertion (3) follows directly from (2) since $\kappa(V)=\operatorname{dim} V$ under the assumption; (2) is due to Iitaka when $\operatorname{ch} k=0$, which generalizes the classical result that, if $\operatorname{dim} K / k=1$ and the genus of $K / k$ is 1 , then the group of all automorphisms of $K / k$ that leaves a given place of $K / k$ fixed is finite. Finally (1) is well known when $K / k$ has a nonsingular complete model.

\section{BIBLIOGRAPHY}

1. S. Iitaka, On logarithmic Kodaira dimension of algebraic varieties, Complex Analysis and Algebraic Geometry, Iwanami, 1977.

2. Z. Luo, Kodaira dimension of algebraic function fields, Amer. J. Math. 109 (1987), $669-694$.

3. , Ramification divisor of regular schemes, preprint.

4. T. Luo and Z. Luo, Factorization of birational morphisms with fiber dimension bounded by 1, Math. Ann. (to appear).

5. H. Stichtenoth, Über das Geschlecht eines inseparablen Funktionenkörpers, Manuscripta Math 14 (1974), 173-182.

ACademia Sinica, Institute of Systems Science, Beijing 100080, China 
